\title{
LES FACTEURS PREDECTIFS DES ECHECS DE LA PHARYNGOTOMIE AU COURS DE LA RHONCHOPATHIE CHRONIQUE
}

\author{
S. SAHTOUT, L. OUERTATANI, S. TRABELSI, R. LAHIANI, S. KHARRAT, N. BELTAIEF, \\ G. BESBES, S. HACHICHA \\ SERVICE ORL ET CHIRURGIE MAXILLO-FACIALE \\ HÔPITAL LA RABTA
}

\begin{abstract}
RESUME
La pharyngotomie est un geste chirurgical actuellement bien codifié dans le traitement de la rhonchopathie chronique. Le but de cette étude rétrospective est de préciser la place de la pharyngotomie dans le traitement de la rhonchopathie chronique par une analyse statistique des résultats cliniques tout en étudiant les facteurs pronostiques influençant les résultats de cette technique chirurgicale.

Cette étude concerne 94 patients ayant tous bénéficiés d'une pharyngotomie chirurgicale : un premier groupe de 80 patients présentant une rhonchopathie simple, et un deuxième groupe de 14 patients présentant un syndrome d'apnée du sommeil (SAS).

La prédominance masculine était nette. L'âge moyen était de 42 ans.

Un examen polysomnographique a été réalisé soit directement, en cas de forte suspicion d'apnée du sommeil, soit en examen de certitude après dépistage.

Le recul moyen était de 20 mois, avec des extrêmes de 1 mois à 12,8 ans.

Après analyse statistique, les facteurs de mauvais pronostic à court terme étaient l'absence d'anomalies amygdaliennes préopératoires et l'absence d'anomalies uvulaires. A plus long terme, les facteurs de récidive du ronflement sont l'existence d'un SAS et l'absence d'anomalies vélaires.

MOTS CLES : Ronchopathie chronique, pharyngotomie, facteurs prédictifs de l'échec
\end{abstract}

The pharyngotomy is now a well known chirurgical cure of the chronic rhonchopathy.

The objective of our retrospective study is to specify the efficiency of this surgery.

This study has interested 94 patients, who have undergone a chirurgical pharyngotomy: the first group is made of 80 patients having a simple rhonchopathy, and the second group is made of 14 patients with sleep apnea.

The mean age of our patients was 42 years. (sex-ratio:2).

Clinical results were analyzed as possible factors predicting success or failure.

After a mean follow-up of 20 months, most patients reported improvement in quality of life.

KEY WORDS : Chronic rhonchopathy, pharyngotomy, factors predicting failure

\section{INTRODUCTION}

La rhonchopathie chronique est à ce jour une entité pathologique bien connue. Elle regroupe toutes les formes cliniques des maladies ayant en commun un signe fonctionnel constant : le ronflement. Liée à une augmentation anormale des résistances des voies aériennes supérieures pendant le sommeil, la rhonchopathie chronique regroupe trois entités : le ronflement simple, qui est la forme la plus bénigne, le syndrome d'apnée du sommeil, qui est la forme la plus grave et le syndrome des résistances anormalement élevées des voies aériennes supérieures, forme plus récemment décrite.

L'étude clinique et surtout l'étude du sommeil par l'enregistrement simultané permettent de distinguer les différentes entités de la rhonchopathie chronique.

La pharyngotomie conventionnelle constitue pour certaines équipes l'intervention de première intention dans la rhonchopathie avec ou sans apnée.

Nous nous proposons dans ce travail d'analyser les différents facteurs pronośtiques pouvant influencer les résultats de la pharyngotomie que ce soit à court terme ou à long terme.

\section{MATÉRIEL ET MÉTHODES}

Cette étude rétrospective a inclus 94 patients traités pour une rhonchopathie chronique dans le service d'ORL et de CMF de la Rabta entre 1987 et 2004.

Tous les patients ont bénéficiés d'une pharyngotomie plus ou moins associée à d'autres gestes, en fonction de l'examen clinique initial.

Nous avons exclu de notre étude :

- Les patients suivis à la consultation, non opérés, qui ont été adressés à d'autres spécialistes pour un traitement médical ou instrumental,

- Les patients opérés pour rhonchopathie chronique, ayant bénéficié d'un geste ne comprenant pas de pharyngotomie telle une amygdalectomie isolée ou une septoplastie,

- Et les malades ayant eu une pharyngotomie qui ne se sont jamais présentés au contrôle médical post-opératoire.

Le bilan préopératoire standardisé a toujours comporté : *un questionnaire d'évaluation clinique recherchant les antécédents, les facteurs de risques exogènes, les symptômes diurnes et nocturnes évocateurs de SAS ainsi que 
le retentissement de la rhonchopathie sur la qualité du sommeil et l'activité diurne.

La somnolence diurne a été jugée excessive lorsqu'elle affectait fréquemment des activités banales (devant la télévision, en post prandial), ou lorsqu'elle affectait des activités moins banales (au volant, au travail).

*un examen ORL complet systématique avec une évaluation précise :

-de la morphologie maxillo-faciale à la recherche d'un syndrome malformatif ou d'une morphologie évocatrice d'une rhonchopathie chronique (cou court, face large, petite bouche), d'un rétrognatisme ou d'un prognatisme ; -de la morphologie du voile du palais,

-du volume amygdalo-lingual,

-du degré d'obstruction nasale.

Une polysomnographie a été réalisée chez 94 patients.

Nous avons retenu le diagnostic de :

- Ronflement simple chez 75 patients,

- Syndrome de résistance des voies aériennes supérieures (SHRVS) chez 5 patients,

- Et de SAS chez 14 patients.

Tous les patients ont bénéficié d'une pharyngotomie chirurgicale associée à une amygdalectomie première chez les patients présentant une hypertrophie amygdalienne importante ou modérée (dans 85 cas) et à une septoplastie dans un cas. Une association des trois gestes a été réalisée dans 6 cas. (tableau I).

\begin{tabular}{|l|l|}
\hline Geste chirurgical & Nombre de patients \\
\hline Pharyngotomie & $2 / 94$ \\
\hline Pharyngotomie+amygdalectomie & $85 / 94$ \\
\hline Pharyngotomie+septoplastie & $1 / 94$ \\
\hline Pharyngotomie+amygdalectomie+septoplastie & $6 / 94$ \\
\hline
\end{tabular}

Tableau I : Geste chirurgical réalisé

Une surveillance post -opératoire stricte de $24 \mathrm{H}$ a été imposée à tous les patients.

Les soins post- opératoires ont comporté une antibioprophylaxie pendant 8 jours associée à des anti-inflammatoires en l'absence de contre-indications.

Les résultats post-opératoires ont été appréciés sur des critères subjectifs tenant compte du devenir du ronflement, des effets indésirables, de la somnolence diurne et de la qualité du sommeil.

Aucun patient n'a bénéficié en post opératoire d'une polysomnographie vu le coût et le manque de disponibilité de cet examen. Par conséquent nous ne disposons pas de moyen objectif pour évaluer l'efficacité de la pharyngotomie sur le SAS.

\section{RÉSULTATS}

Population 1 : Rhonchopathie simple (ronfleurs simples ou présentant un SHRVS)

Cette population comporte 80 patients $(54 \mathrm{H}$ et $26 \mathrm{~F})$. Le sex-ratio étant de 2,07.
L'âge moyen était de 42 ans avec des extrêmes allant de 27 à 70 ans. La médiane était de 40 ans.

La surcharge pondérale a été notée chez $44 \%$ de cette population.

Une pharyngotomie associée à une amygdalectomie a été pratiquée chez 73 patients, une pharyngotomie seule chez un patient et une pharyngotomie associée à une septoplastie chez un patient. Un protocole complet associant une pharyngotomie, une amygdalectomie et une septoplastie a été effectuée chez 5 patients.

Tous les patients opérés se sont présentés à un mois de l'intervention.

Soixante trois patients signalaient à un mois une disparition du ronflement. Ce dernier a été remplacé par un bruit moins gênant et perturbant beaucoup moins le sommeil chez 11 patients. Les six patients restants n'ont éprouvé aucune amélioration.

Les résultats à un mois ont été jugés bons pour $92,5 \%$ des patients et mauvais pour $7,5 \%$ des patients.

Les résultats à plus long terme variaient en fonction du délai post-opératoire (Figure 1).

A un mois de l'intervention, le resultat a été jugé satisfaisant chez 74 patients et mauvais chez 6 patients.

A 2 ans de l'intervention et sur un total de 19 patients suivis régulièrement, le résultat a été jugé bon chez 15 patients et mauvais chez 4 patients.

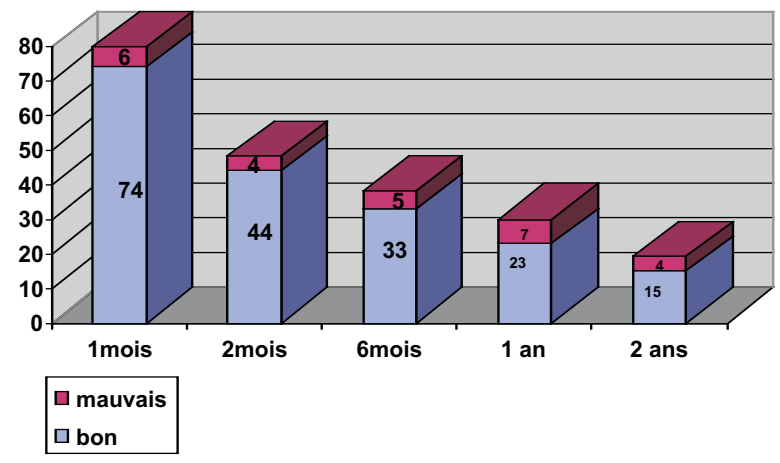

Fig. 1: Résultats post-opératoires (population1)

Globalement, sans tenir compte de l'importance du délai, 65 patients se disent satisfaits de l'intervention.

\section{Population 2 : Syndrome d'apnée du sommeil}

Cette population comprend 14 patients $(11 \mathrm{H}$ et $3 \mathrm{~F})$; le sex ratio était de 3,66 .

L'âge moyen était de 41,8 ans avec des extrêmes de 26 à 67 ans.

Une surcharge pondérale a été notée chez 10 patients. Tous les patients ont bénéficiés d'un enregistrement du sommeil préopératoire : il s'agissait d'une polysomnographie dans 12 cas, d'une polygraphie à 4 chaînes dans un cas et de respisomnographie dans un autre cas.

Lindex de perturbations respiratoires (IAH) était supérieur à 10 dans tous les cas. 
La saturation moyenne nocturne en oxygène était de 93,4\% (extrêmes : $87 \%$ et $97 \%$ ).

Une pharyngotomie seule a été réalisée chez un seul patient. Une pharyngotomie associée à une amygdalectomie a été pratiquée chez 12 patients. Un protocole complet comportant une pharyngotomie, une amygdalectomie et une chirurgie nasale a été effectuée chez un patient. Le geste chirurgical a été précédé d'une cure d'amaigrissement chez 7 patients.

A un mois de l'intervention douze patients signalaient une nette régression du ronflement.

Deux patients ne signalaient aucune amélioration.

Les résultats à un mois ont été jugés bons chez $85,7 \%$ des patients et mauvais chez $14,3 \%$ des patients.

Les résultats à plus long terme sont résumés dans l'histogramme suivant (Figure 2).

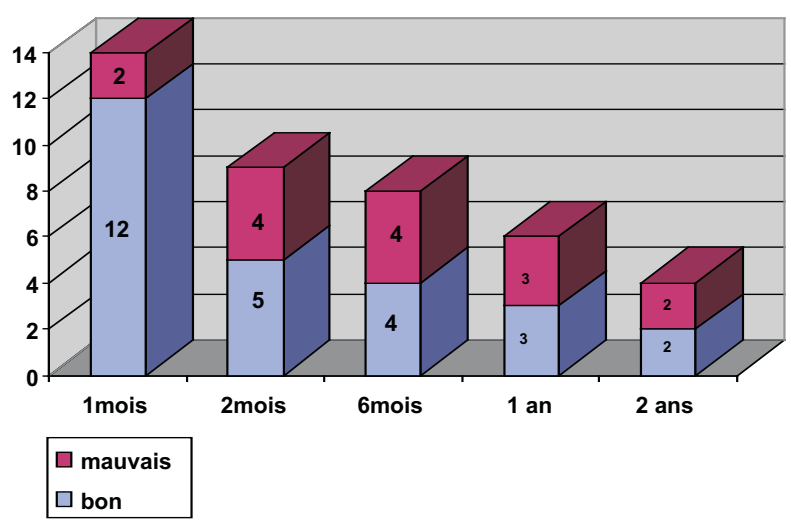

Fig. 1: Résultats post-opératoires (population 2)

Globalement, sans tenir compte de l'importance du délai, huit patients se disent satisfaits de l'intervention.

De manière subjective, la somnolence diurne était une plainte rapportée par 29 patients, de l'ensemble des populations 1 et 2 avant l'intervention. Parmi ces patients, $16(55 \%)$ ont déclaré une disparition ou une amélioration de ce symptôme. Une amélioration partielle suivie d'une récidive a été rapportée dans 6 cas $(21 \%)$. Aucune amélioration n'a été notée chez 2 patients $(7 \%)$. L'évolution n'a pas été précisée chez 5 patients (17\%).

Aucune complication péri-opératoire n'a été observée.

Quatre patients ont présenté un rétrécissement partiel de l'isthme nasopharyngé avec pour conséquence, une réapparition du ronflement. Un patient a été repris chirurgicalement. II a eu une pharyngotomie d'élargissement. Une analyse statistique à la recherche de facteurs prédictifs d'échec ou de succès de la pharyngotomie a été réalisée. Plusieurs facteurs ont été étudiés dans cet objectif (tableau II).

\begin{tabular}{|c|c|c|}
\hline Facteur & $\begin{array}{c}\text { Bons } \\
\text { résultats en \% } \\
(1 \text { mois })\end{array}$ & $\begin{array}{l}\text { P (Signification } \\
\text { statistique) }\end{array}$ \\
\hline $\begin{array}{r}\text { Obésité (IMC>27): oui }(\mathrm{n}=46) \\
\text { non }(\mathrm{n}=48)\end{array}$ & \begin{tabular}{l|l}
89,1 & \\
93,8 & \\
\end{tabular} & $\begin{array}{c}0,5 \\
\text { Non significatif }\end{array}$ \\
\hline $\begin{array}{r}\text { Type de rhonchopathie: SAS }(\mathrm{n}=14) \\
\operatorname{RS}(\mathrm{n}=80)\end{array}$ & $\begin{array}{l}85,7 \\
92,5\end{array}$ & $\begin{array}{c}0,34 \\
\text { Non significatif }\end{array}$ \\
\hline $\begin{array}{r}\text { Cou court : oui }(\mathrm{n}=35) \\
\text { non }(\mathrm{n}=59)\end{array}$ & $\begin{array}{l}88,6 \\
93,2\end{array}$ & $\begin{array}{c}0,5 \\
\text { Non significatif }\end{array}$ \\
\hline $\begin{array}{l}\text { Anomalies amygdaliennes et vélopharyngées: } \\
\text { oui }(n=84) \\
\text { non }(n=9)\end{array}$ & $\begin{array}{l}95,2 \\
66,7\end{array}$ & $\begin{array}{c}0,0018 \\
\text { significatif }\end{array}$ \\
\hline $\begin{array}{l}\text { Amygdales hypertrophiées ou enchatonnées: } \\
\text { oui }(n=85) \\
\text { non }(n=9)\end{array}$ & $\begin{array}{l}94,1 \\
66,7\end{array}$ & $\begin{array}{c}\mathbf{0 , 0 2 7} \\
\text { significatif }\end{array}$ \\
\hline $\begin{array}{r}\text { Luette longue et/ou épaisse: oui }(\mathrm{n}=87) \\
\text { non }(\mathrm{n}=7)\end{array}$ & $\begin{array}{l}\mathbf{9 4 , 3} \\
\mathbf{5 7 , 1}\end{array}$ & $\begin{array}{c}0,012 \\
\text { significatif }\end{array}$ \\
\hline $\begin{array}{r}\text { Voile flasque : oui }(\mathrm{n}=76) \\
\text { non }(\mathrm{n}=18)\end{array}$ & $\begin{array}{l}93,4 \\
83,3\end{array}$ & $\begin{array}{c}0,18 \\
\text { Non significatif }\end{array}$ \\
\hline $\begin{array}{l}\text { Déviation septale et/ou hypertrophie turbinale: } \\
\text { oui }(\mathrm{n}=36) \\
\text { non }(\mathrm{n}=58)\end{array}$ & $\begin{array}{l}83,3 \\
96,6\end{array}$ & $\begin{array}{c}0,051 \\
\text { Proche de la } \\
\text { signification }\end{array}$ \\
\hline
\end{tabular}

Tableau II : Facteurs prédictifs des échecs de la pharyngotomie

Parmi les facteurs étudiés les anomalies amygdaliennes et vélopharyngées, les amygdales hypertrophiées ou enchatonnées ainsi que les luettes longues et/ou épaisses ont représentée des facteurs de risque significatifs au cours de la rhonchopathie chronique.

La déviation septale et l'hypertrophie turbinale ont représenté des facteurs proche de la signification.

\section{DISCUSSION}

La nature pathologique du ronflement simple est l'objet de controverses. Pendant longtemps, le ronflement a été considéré comme un phénomène normal, objet de plaisanteries. Au mieux il était considéré comme une nuisance familiale et sociale (7).

Le syndrome d'apnée du sommeil, est quant à lui, reconnu comme un facteur de morbidité et de mortalité.

La rhonchopathie chronique est à ce jour une entité pathologique bien connue, mais dont le traitement demeure délicat et sujet à caution.

Les facteurs de risque les plus clairement identifiés du SAS et de la rhonchopathie simple sont l'obésité, les rétrécissements oropharyngés, les anomalies anatomiques cervico-faciales, le sexe masculin et l'âge avancé. Le but du traitement dans le ronflement simple est la suppression de la gêne sociale provoquée par le ronflement bruit.

Dans le SAS, l'objectif est de corriger les symptômes nocturnes et diurnes, mais également de supprimer le risque de complications.

De nombreux traitements ont été proposés dans la rhonchopathie chronique et font appel à des techniques très diverses allant du simple traitement médical, aux techniques chirurgicales les plus complexes, en passant par le traitement instrumental par orthèses ou par ventilation par pression positive continue par voie nasale.

La pharyngotomie représente un des moyens thérapeutiques les plus simples et efficaces de la rhonchopathie chronique.

Cette intervention consiste grossièrement à diminuer l'excès muqueux au niveau du voile et des parois latérales de l'oropharynx et d'induire une rigidification des parois oro- 
pharyngées par un processus de cicatrisation.

Les complications de la pharyngotomie sont de trois ordres (6) :

- les complications vitales péri-opératoires (hémorragie, majoration de l'obstruction des voies aéro-digestives supérieures),

- les complications fonctionnelles mineures (douleur postopératoire, surinfection, reflux nasopharyngé transitoire, troubles du goût, troubles de la parole, paresthésies pharyngées),

- les complications fonctionnelles majeures dominées par l'insuffisance vélaire et les sténoses nasopharyngées.

Les résultats de la pharyngotomie sont appréciés en fonction du stade de la rhonchopathie chronique.

Dans la rhonchopathie chronique simple, sans apnée du sommeil, l'appréciation des résultats fait appel, dans la plupart des études à des éléments purement subjectifs (concernant essentiellement l'évolution du ronflement) par l'intermédiaire de questionnaires. Il est en effet extrêmement complexe de définir un ronflement sur des critères objectifs d'énergie et de fréquence. De plus, on ne retrouve pas de corrélation entre les appréciations subjectives et objectives du ronflement.

Le taux de succès (disparition complète du ronflement ou diminution compatible avec la satisfaction du conjoint) est compris entre 76 et $87 \%$, suivant les auteurs (7). II a été de $81 \%$ dans notre étude.

Dans le syndrome d'apnée du sommeil, les résultats de la pharyngotomie peuvent être évalués selon des critères subjectifs, mais également sur des critères objectifs.

On retrouve selon les séries une amélioration du ronflement (diminution ou disparition) dans 37 à $97 \%$ des cas $(4,5,8)$. Cette amélioration était de $57 \%$ dans notre série. On retrouve également une diminution, voire une disparition de la somnolence diurne dans 52 à $90 \%$ des cas (16). Une régression de la somnolence diurne a été signalée par $55 \%$ des patients dans notre étude. II existe également une amélioration non négligeable des autres troubles fonctionnels, comme l'asthénie matinale, les céphalées (16). Une amélioration de la qualité du sommeil a été observée dans $69 \%$ des cas (8).

Ces résultats peuvent être discutés, car il s'agit essentiellement d'appréciation subjective. L'absence d'étude contrôlée ne permet pas d'exclure un effet placebo de la chirurgie.

La polysomnographie permet une étude objective de l'efficacité de la pharyngotomie. Le taux de succès, apprécié par un enregistrement du sommeil réalisé au moins trois mois après l'intervention, est compris entre 32 et $80 \%$. II est différemment apprécié selon la variable polysomnographique retenue comme critère d'évaluation.

Plusieurs auteurs ont observé une diminution du taux d'efficacité de la pharyngotomie avec le temps. Dans notre série le taux de succès à un mois était de $92,5 \%$ dans la population de rhonchopathes simples et de $86 \%$ en cas de syndrome d'apnée du sommeil. Après un délai de 2 ans, il passe respectivement à $79 \%$ et à $50 \%$.
Toutefois le résultat observé est sûrement biaisé par le nombre de perdus de vue.

Les principaux facteurs péjoratifs rapportés par la majorité des auteurs sont le poids, les apnées et l'obstruction rétrobasilinguale $(2,3,4,17)$.

L'obésité semble franchement de mauvais pronostic dès que l'index de masse corporelle dépasse 27 ou 28 ou que le poids dépasse $130 \%$ de la valeur théorique (3). Dans notre série, l'effet péjoratif de l'obésité n'a pas été objectivé par l'étude statistique.

Un index d'apnée élevé est aussi un facteur prédictif puissant d'échec de la pharyngotomie. Le taux d'échec apparaît très étroitement corrélé à l'index d'apnée préopératoire.

Dans notre étude, les résultats obtenus sont meilleurs en cas de rhonchopathie simple comparés à ceux obtenus en cas de SAS (taux d'efficacité respectifs de 81 et $57 \%$ ). L'indication d'une pharyngotomie isolée semble par conséquent déraisonnable pour un ronfleur présentant un SAS sévère $(3,4)$. La limite supérieure à fixer n'est pas unanimement appréciée. Elle est de 30 apnées / hypopnées par heure de sommeil pour certains auteurs et de 50 pour d'autres $(2,12)$.

La multiplicité des sites obstructifs, notamment la présence d'un obstacle rétrobasilingual associé serait un facteur prédictif de mauvais résultat.

Plusieurs auteurs ont essayé d'apprécier le site d'obstruction en pré-opératoire afin d'améliorer l'efficacité de cette technique par une meilleure sélection des patients (examen clinique, céphalométrie, IRM, TDM, mesure des pressions pharyngées...).

Dans notre étude, l'examen clinique a été notre seule exploration anatomique des voies aériennes supérieures. Ces données permettent donc de limiter les indications de la pharyngotomie à des patients souffrant d'une rhonchopathie simple ou d'un SAS peu sévère :

- dont l'IAH est inférieur à 30 par heure de sommeil,

- dont l'IMC est inférieur à $30 \mathrm{Kg} / \mathrm{m} 2$,

- dont l'anomalie vélaire est patente et parait isolée (1),

- chez lesquels un bilan morphologique n'a pas montré d'anomalies osseuses.

D'autres facteurs ont été évoqués mais restent controversés.

Pour certains auteurs, l'âge avancé est plus pourvoyeur d'échecs.

L'influence de la consommation d'alcool et de l'intoxication tabagique est diversement appréciée.

L'ensemble de ces critères de sélection ne possède qu'une valeur relative, car certains patients très obèses, avec des index d'apnée élevés, ont pu bénéficier avec succès de cette chirurgie. (Tableau III) 


\begin{tabular}{|l|c|c|c|c|}
\hline Référence & n & Age & IMC & Sévérité du SAS \\
\hline Dondar 1997 (8) & 37 & Jeune age & Non obèse & IAH \\
\hline Lévy 1997 & 26 & Non prédictif & Non prédictif & Non prédictif \\
\hline Rampelberg 1993 & 20 & Non prédictif & Non prédictif & Non prédictif \\
\hline Chabolle 1995 (4) & 150 & Non prédictif & IMC $<27$ & IAH<30 \\
\hline Larson 1994 (12) & 50 & Non prédictif & IMC $<30$ & IAH $<50$ \\
\hline
\end{tabular}

IAH : Index d'apnée hypopnée

IMC : Indice de masse corporelle

Tableau III : Critères de sélection des «bons» candidats à la pharyngotomie (âge, IMC, IAH)

\section{CONCLUSION}

La prise en charge et le traitement de la rhonchopathie chronique reste un parcours complexe.

La pharyngotomie isolée proposée en première intention à l'issue d'un enregistrement polysomnographique est une option thérapeutique simple, efficace et peu coûteuse pour les rhonchopathes simples et les SAS légers sans surcharge pondérale majeure.

Pour les SAS plus sévères, il apparaît déraisonnable de proposer une pharyngotomie isolée.

\section{REFERENCES}

1- Bettega G, Verain A, Pépin JL, Lévy P, Raphael B. Conduite à tenir thérapeutique devant un syndrome d'apnées obstructives du sommeil : la place du stomatologiste. Rev Stomatol Chir Maxillofac. 2002 ; $103: 188-9$.

2- Chabolle F, De Dieuleveult T, Cabanes $\mathrm{J}$ et al. Résultats à long terme de la pharyngotomie chirurgicale classique (Uvulo-palato-pharyngoplastie) versus laser $\mathrm{CO} 2$ en ambulatoire dans le traitement de la rhonchopathie simple. Ann Otolaryngol Chir Cervicofac. $1998 ; 115: 196-201$.

3- Chabolle F, Fleury B, De Corbiere S, Pandraud L, Freche $\mathrm{CH}$. Le traitement chirurgical du syndrome d'apnée du sommeil. Rev Mal Resp 1990, 7:451-8.

4- Chabolle F, Sequert S, Gleury B et al. Analyse des résultats de la pharyngotomie dans le traitement chirurgical du syndrome d'apnées du sommeil. A propos de 150 cas. Ann Otolaryngol Chir Cervicofac 1995, $112: 98-106$.

5- Chevalier D, Robert Y, Mllart A, Quieu JD, Piquet JJ. Traitement chirurgical du ronflement et des apnées du sommeil. Résultats à propos de 59 patients. Ann Otolaryngol Chir Cervicofac 1994, 111 : 393-6.

6 - Dejean Y, Chouard CH. La rhonchopathie chronique. Rontlement et syndrome d'apnées du sommeil. Société française d'olo-rhino-laryngologie et de pathologie cervico-faciale. Paris. Arnette 1993.

7- Dejean $Y$, Crampette L. Insuffisance respiratoire d'origine pharyngée (inonchopathie et syndrome d'apnées au cours du sommeil). Encycl Med Chir OtoRhino-Laryngologie $1992 ; 20-261$ - A10 :10p.

8- Dondar A, Gerek M, Ozunlu A, Yetiser S. Patient selection and surgical results in obstructive sleep apnea: Eur Arch Otorhinolaryngol Suppl 1997, 254:S157-61. 9- Esclamado RM, Glenn MG, Mc Culloch TM, Cummings CW. Péri-opérative complications and risk factors in the surgical treatment of obstructive sleep apnea syndrome. Laryngoscope 1989, 99: 1125-9.
10- Fleury B, Chabolle F, Laffont $F$ et al. Effets à 3 mois de l'uvulopalatopharyngoplastie dans le traitement du syndrome d'apnées obstructives du sommeil de l'adulte. Rev Mal Resp 1989, $6: 519-24$.

11- Katsantonis GP, Friedman WH, Krebs FJ, Walsh JK. Nasopharyngeal complications following uvulopalatopharyngoplasty. Laryngoscope 1987, 97: 309-14. 12- Larsson LH, Garlsson-Nordlander B, Svanborg B. Four-year follow-up after uvulopalatopharyngoplasty in 50 unselected patients with obstructive sleep apnea syndrome. Laryngoscope 1994, 104: 1362-8.

13- Makeieff $M$, Crampette $L$. Les sténoses cicatricielles vélo-pharyngées. Physiopathologie, clinique, traitement. Les cahiers d'ORL 2002,34:306-11.

14- Meyer B, Chabolle F, Chouard CH. Résultats et complications à long terme de l'uvulopalatopharyngoplastie dans la rhonchopathie sans syndrome d'apnées du sommeil. Ann Otolaryngol $1988 ; 105: 291-7$.

15- Meyer B, Chabolle F, Chouard CH. Les traitements chirurgicaux de la rhonchopathie chronique: description et indications. Ann Otolaryngol 1988; 105 : 277-282.

16- Pessey J. Pharyngotomie : technique, résultats, indications. Les cahiers d'ORL $1997 ; 32: 419-24$

17-Abdelkefi M., Harzallah M., Belakhdhar M. et al. Ronchopathie chronique de l'adulte J. Tun. ORL. 2006; 16: 1-4 